\title{
Esculetin, a coumarin derivative, exerts in vitro and in vivo antiproliferative activity against hepatocellular carcinoma by initiating a mitochondrial-dependent apoptosis pathway
}

\author{
J. Wang ${ }^{1,2}$, M.L. Lu ${ }^{2}$, H.L. Dai ${ }^{2}$, S.P. Zhang ${ }^{2}$, H.X. Wang ${ }^{2}$ and N. Wei ${ }^{1}$ \\ ${ }^{1}$ The First Affiliated Hospital, Liaoning Medical University, Jinzhou, China \\ ${ }^{2}$ Key Laboratory of Cardiovascular and Cerebrovascular Drug Research, Liaoning Province, Liaoning Medical University, \\ Jinzhou, China
}

\begin{abstract}
This study investigated the in vitro and in vivo antiproliferative activity of esculetin against hepatocellular carcinoma, and clarified its potential molecular mechanisms. Cell viability was determined by the MTT (tetrazolium) colorimetric assay. In vivo antitumor activity of esculetin was evaluated in a hepatocellular carcinoma mouse model. Seventy-five C57BL/6J mice were implanted with Hepa1-6 cells and randomized into five groups $(n=15$ each) given daily intraperitoneal injections of vehicle (physiological saline), esculetin $\left(200,400\right.$, or $\left.700 \mathrm{mg} \cdot \mathrm{kg}^{-1} \cdot \mathrm{day}^{-1}\right)$, or $5-\mathrm{Fu}\left(200 \mathrm{mg} \cdot \mathrm{kg}^{-1} \cdot \mathrm{day}^{-1}\right)$ for 15 days. Esculetin significantly decreased tumor growth in mice bearing Hepa1-6 cells. Tumor weight was decreased by 20.33 , 40.37, and $55.42 \%$ with increasing doses of esculetin. Esculetin significantly inhibited proliferation of HCC cells in a concentration- and time-dependent manner and with an $\mathrm{IC}_{50}$ value of $2.24 \mathrm{mM}$. It blocked the cell cycle at $\mathrm{S}$ phase and induced apoptosis in SMMC-7721 cells with significant elevation of caspase- 3 and caspase- 9 activity, but did not affect caspase- 8 activity. Moreover, esculetin treatment resulted in the collapse of mitochondrial membrane potential in vitro and in vivo accompanied by increased Bax expression and decreased Bcl-2 expression at both transcriptional and translational levels. Thus, esculetin exerted in vitro and in vivo antiproliferative activity in hepatocellular carcinoma, and its mechanisms involved initiation of a mitochondrial-mediated, caspase-dependent apoptosis pathway.
\end{abstract}

Key words: Esculetin; Apoptosis; Hepatocellular carcinoma; Mitochondrial-dependent pathway; Antitumor activity

\section{Introduction}

Hepatocellular carcinoma (HCC) is the fifth most common cancer and the second leading cause of cancerrelated deaths worldwide (1). The evidence suggests that the incidence of $\mathrm{HCC}$ is rising, making it a major health problem, especially in China, where the incidence of HCC has increased by $50 \%$ over the past 10 years (2). The current treatments for HCC are surgery and chemotherapy. However, most HCC patients are not candidates for surgical resection because, at the time of diagnosis, the tumor may be too large or has expanded into nearby major blood vessels or metastasized (3). In addition, the efficacy of chemotherapy is not high, and the side effects are often difficult to tolerate. Thus, novel anticancer therapeutic agents for treatment of HCC are urgently needed.
Natural products have been a primary source of discovery and development of anticancer drugs and novel natural products that have antitumor activity against HCC may be found. Esculetin, a phenolic compound that occurs in various plants, has displayed beneficial pharmacological and biochemical properties including anticancer, antiinflammatory, neuroprotective, and antioxidant activity (4-8). Esculetin was shown to have anticancer activity in human colon cancer, breast cancer, human leukemia, and cervical cancer, and to inhibit cell proliferation in human colon cancer through the Ras/ERK1/2 pathway (9). Park et al. (10) reported that esculetin suppressed the c-Jun $\mathrm{N}$-terminal kinase (JNK) and extracellular-signal-regulated kinase (ERK) pathways, and induced apoptosis in human 
leukemia U937 cells. In addition, esculetin was shown to sensitize HepG2 cells to the effects of taxol (11). However, in vitro and in vivo studies have not yet confirmed the therapeutic effect of esculetin on growth of HCC or revealed its molecular mechanisms of action.

This study evaluated the in vivo and in vitro antitumor effect of total alkaloids of esculetin in a mouse xenograft model and in cultures of SMMC-7721 hepatocellular carcinoma cells. The potential mechanism of esculetin against $\mathrm{HCC}$ is also described.

\section{Material and Methods}

\section{Reagents}

The isolation, identification, and purity assessment of esculetin were performed in our laboratory as previously described (12). 5-Fu was obtained from Tianjing Jinyao Anjisuan Medicine Co., Ltd. (China). The Annexin V-FITC apoptosis detection kit was purchased from Beijing Biosea Biotechnology Co., Ltd. (China). Propidium iodide (PI), dimethyl sulfoxide (DMSO), and 3-(4,5-dimethylthiazol)-2, 5-diphenyltetrazolium bromide (MTT) were obtained from Amresco (USA). $\beta$-actin, caspase-3/-9, Bcl-2 and Bax antibodies were from Beijing Biosynthesis Biotechnology (China). 5,5',6,6'-tetrachloro-1,1',3,3'-tetraethyl-benzimidazol-carbocyanine iodide (JC-1) and the RT-PCR kit were obtained from Beyotime Institute of Biotechnology (China). Dulbecco's modified Eagle's medium (DMEM) was from Gibco (USA) and trypsin from Hyclone (USA).

\section{Animals}

The experimental protocols were approved by the ethics committee of Liaoning Medical College for the use of experimental animals for research and teaching. Seventyfive 6-week-old C57BL/6J male mice (20-22 g) were purchased from the Animal Centre of Chinese Medical University. All mice were housed in a specific-pathogen free laboratory. The mice were fed standard rodent pellets and allowed free access to filtered water. All experimental procedures were performed in accordance with the Guidelines of Animal Experiments from the Committee of Medical Ethics, National Health Department of China.

\section{Animal tumor model}

The Hepa1-6 cell line was purchased from the Type Culture Collection of the Chinese Academy of Sciences, Shanghai, China. Cells were cultured in DMEM medium with $10 \%$ fetal calf serum (FCS) and 90\% DMEM and maintained in a humidified atmosphere of $5 \% \mathrm{CO}_{2}$ at $37^{\circ} \mathrm{C}$. Hepa1-6 cells were inoculated subcutaneously in the right axilla of C57BL/6J mice $\left(1 \times 10^{7}\right.$ viable cells $\left./ \mathrm{mL}\right)$ to establish a xenograft model. Three days after implantation, mice were randomized into 5 groups $(n=15$ each) and injected intraperitoneally with vehicle (physiological saline), esculetin $\left(200,400\right.$, or $700 \mathrm{mg} \cdot \mathrm{kg}^{-1} \cdot$ day $\left.^{-1}\right)$, or $5-\mathrm{Fu}\left(200 \mathrm{mg} \cdot \mathrm{kg}^{-1} \cdot\right.$ day $\left.^{-1}\right)$ as a positive control for 15 days. In vivo antitumor activity of esculetin was evaluated by weight and inhibition rate. Tumor inhibition (\%) was measured by the following ratio: $\left(1-\mathrm{W}_{\text {Treated }}\right) / \mathrm{W}_{\text {Control }} \times 100 \% \mathrm{~W}_{\text {Treated }}$ and $\mathrm{W}_{\text {Control }}$ were the average tumor weights in treated and control mice.

\section{Cell culture and viability assay}

The SMMC-7721 human hepatocellular carcinoma cell line was obtained from the Scientific Experiment Center of Liaoning Medical College (China) and maintained at $37^{\circ} \mathrm{C}$ in humidified $95 \%$ air and $5 \% \mathrm{CO}_{2}$ in DMEM supplemented with $10 \%$ heat-inactivated fetal bovine serum (FBS). The cells were subcultured at $80 \%$ confluency.

Cell proliferation was assayed as previously described (13). SMMC-7721 cells were resuspended in DMEM with $10 \%$ FBS and seeded onto 96-well plates at a density of $1 \times 10^{4}$ cells $/ \mathrm{mL}$ in $0.1 \mathrm{~mL}$ medium and cultured for $24 \mathrm{~h}$. The cells were treated with various concentrations of esculetin for 24, 48, and $72 \mathrm{~h}$. At the end of the treatment, $20 \mu \mathrm{L}$ MTT was added to each well, and the cells were incubated for another $4 \mathrm{~h}$. The purple-blue MTT formazan precipitate was dissolved in $150 \mu \mathrm{L}$ DMSO and was measured at $570 \mathrm{~nm}$ using a multi-mode microplate reader (BioTek Instruments, Inc., USA). All measurements were performed three times. Growth inhibition (\%) was measured by the following ratio: $\left(1-A_{\text {Treated }} / A_{\text {Control }}\right) \times 100 \%$. $A_{\text {Treated }}$ and $A_{\text {Control }}$ were the average absorbance of three parallel experiments from treated and control groups. $I_{50}$ was the concentration that caused $50 \%$ inhibition of cell viability and was calculated by the logit model.

\section{Cell cycle analysis}

SMMC- 7721 cells $\left(1 \times 10^{5} / \mathrm{mL}\right.$ cells per culture flask) were incubated for $24 \mathrm{~h}$, and harvested after esculetin (0, $1.12,2.24$, or $4.48 \mathrm{mM})$, or $5-\mathrm{FU}(0.77 \mathrm{mM})$ treatment for $48 \mathrm{~h}$. The cells were washed in cold PBS two or three times and fixed in $70 \%$ ice-cold ethanol at $4{ }^{\circ} \mathrm{C}$ overnight. Cells were then stained with $0.5 \mathrm{mg} / \mathrm{mL}$ PI containing $0.5 \mathrm{mg} / \mathrm{mL}$ RNase and incubated at $4^{\circ} \mathrm{C}$ for $30 \mathrm{~min}$ in the dark. Cell cycle analysis was done by flow cytometry using a FACSCalibur system (Becton Dickinson, USA).

\section{Determination of caspase activity}

Caspase- $3,-9$, and -8 activity induced by esculetin was determined by colorimetric assay kits (Beyotime Institute of Biotechnology) according to the manufacturer's instructions. All measurements were performed 3 times, and caspase activity was determined by measuring changes in absorbance at $405 \mathrm{~nm}$ using an ELISA reader (BioTek Instruments, Inc.).

\section{Apoptosis assay}

Apoptosis of HCC cells was determined by flow cytometry analysis using an annexin assay. For the in vivo assay, tumor tissue was immersed in physiological saline and a cell suspension was prepared in a tissue homogenizer. For the in vitro assay, SMMC-7721 cells were 
harvested as previously described, concentrated, and washed with cold PBS. Cells were stained with Annexin V-FITC using an assay kit (BD Biosciences, USA) according to the manufacturer's instructions. Data were analyzed using the Bioconsort software (USA).

\section{Mitochondrial membrane potential assessment}

Mitochondrial membrane potential (MMP, $\Delta \Psi \mathrm{m})$ was determined by flow cytometric analysis of JC-1 staining. JC1 is a fluorescent dye that reflects changes in MMP in living cells. Mitochondrial injury results in reduced MMP and leads to an increase in green fluorescence. MMP is reported as the ratio of red to green fluorescence intensity. Cells were obtained as previously described and JC-1 (Beyotime Institute of Biotechnology) staining was performed according to the manufacturer's instructions. Relative fluorescence intensities were monitored by flow cytometry (FACSCalibur). Similarly, $200 \mathrm{~g}$ tumor tissue slices obtained from C57BL/6J mice used in the in vivo experiments were homogenized with a glass homogenizer (Haimen Hua Kai Experiment Glass Instrument Co., Ltd., China), and the homogenate was centrifuged at $1500 \mathrm{~g}$ for $10 \mathrm{~min}$. The supernatant was centrifuged again and resuspended with $50 \mu \mathrm{L}$ Store Buffer. Then MMP in tumor tissues was assayed using a biopsy MMP kit (Genmed Scientific, China) and monitored by flow cytometry (FACSCalibur).

\section{Western blot analysis}

Cells were prepared as previously described. After $24 \mathrm{~h}$, cells were harvested, washed with PBS, and lysed in lysis buffer. Lysates were centrifuged at $12,500 \mathrm{~g}$ for $5 \mathrm{~min}$ at $4^{\circ} \mathrm{C}$, and the total protein concentration was measured. The protein samples were fractionated using $10 \%$ sodium dodecyl sulfate-polyacrylamide gel electrophoresis (SDSPAGE) and transferred to polyvinylidene difluoride (PVDF) membranes. The membranes were incubated with primary antibodies for $16 \mathrm{~h}$ at $4^{\circ} \mathrm{C}$, followed by secondary antibodies conjugated to horseradish peroxidase $(1: 1000)$ for $1 \mathrm{~h}$ at $37^{\circ} \mathrm{C}$. $\beta$-Actin was used to normalize protein loading.

Tumor tissue was thawed in lysis buffer and homogenized using a dounce homogenizer (Haimen Hua Kai Experiment Glass Instrument Co., Ltd.). The homogenate was then centrifuged at $12,000 \mathrm{~g}$ for $30 \mathrm{~min}$ at $4^{\circ} \mathrm{C}$. The protein concentration of the supernatants was determined by the Bradford method. Each sample was then loaded into alternate lanes for gel electrophoresis. Membrane transfer was performed and incubated with rabbit antibody against Bax and Bcl-2. $\beta$-Actin was used as the loading control. Images were captured and analyzed with the Quantity One software (Bio-Rad Laboratories, USA).

\section{RT-PCR analysis}

$\mathrm{Bax}$ and $\mathrm{Bcl}-2$ gene expression was detected by RTPCR. Total RNA was extracted from tumor tissues using Trizol according to the manufacturer's instructions (Promega, USA). cDNA was synthesized using SuperScript III Reverse
Transcriptase (Invitrogen, USA). The sequences of the primers used for amplification of Bcl-2, Bax and GAPDH transcripts were as follows: Bax forward, 5'-CCA GGA TGC GTC CAC CAA GAA-3' and reverse, 5'-AGC AAA GTA AAG AGG GCA ACC AC-3'; Bcl-2 forward, 5'-CTC TGG TTG GGA TTC CTA CGG-3' and reverse, 5'-CGG CAT GAT CTT CTG TCA AGT TTA-3'; GAPDH forward, 5'-TG TTC CTA CCC CCA ATG TGT CCG TC-3' and reverse, 5'-CT GGT CCT CAG TGT AGC CCA AGA TG-3'. Initial template denaturation was performed for $5 \mathrm{~min}$ at $95^{\circ} \mathrm{C}$. The cycle profiles were programmed as follows: $2 \mathrm{~s}$ at $95^{\circ} \mathrm{C}$ (denaturation), $20 \mathrm{~s}$ at $60^{\circ} \mathrm{C}$ (annealing), and $30 \mathrm{~s}$ at $72^{\circ} \mathrm{C}$ (extension). The PCR reaction products were applied to a $2 \%$ agarose gel and separated by electrophoresis for
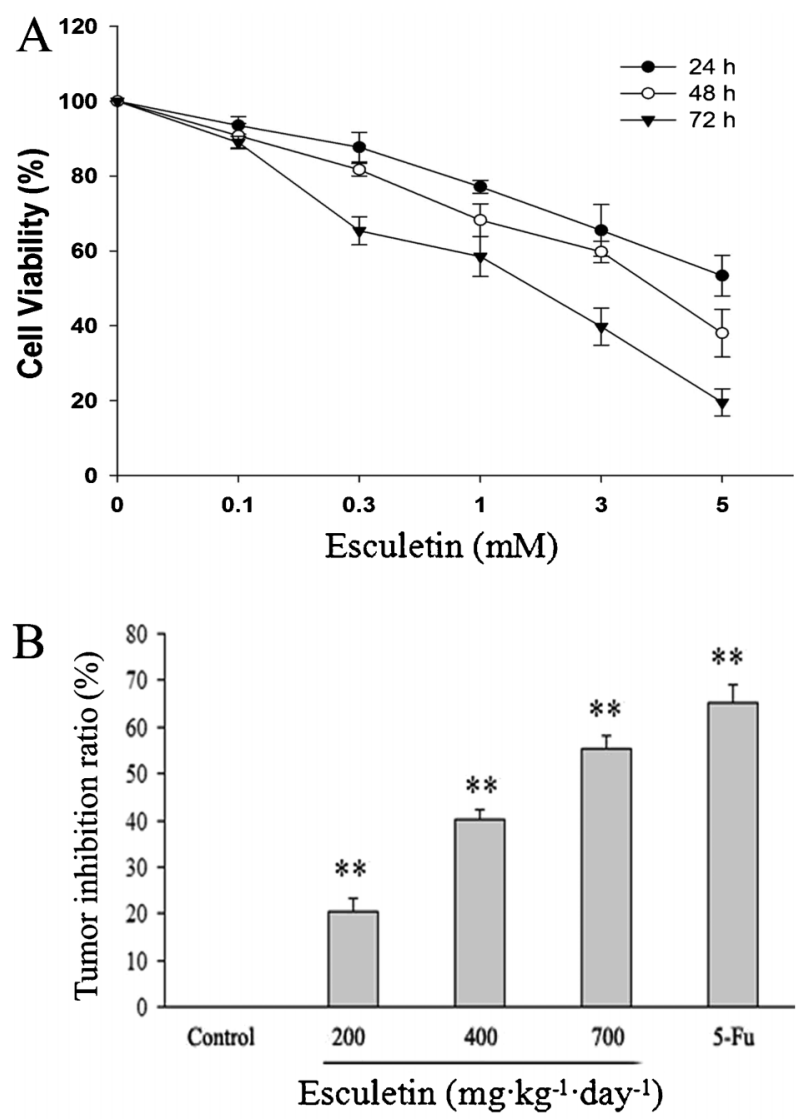

Figure 1. Effect of esculetin on the growth of hepatocellular carcinoma in vivo and in vitro. SMC7721 cells were seeded onto 96-well plates, and then treated with different concentrations of esculetin for 24,48 , and $72 \mathrm{~h}$. Cell viability was determined by MTT assay $(A)$. Hepa1-6 cells were inoculated subcutaneously at the right axilla of $\mathrm{C} 57 \mathrm{BL} / 6 \mathrm{~J}$ mice $\left(1 \times 10^{7}\right.$ viable cells $\left./ \mathrm{mL}\right)$ to establish the xenograft model. Tumor inhibition $(B)$ is reported as a ratio: tumor inhibition $(\%)=\left(1-W_{\text {Treated }}\right) / W_{\text {Control }} \times 100 \%$. $\mathrm{W}_{\text {Treated }}$ and $\mathrm{W}_{\text {Control }}$ are the average tumor weights in treated and control mice, respectively. Data are reported as means $\pm S D$ for $n=15$. ${ }^{* *} \mathrm{P}<0.01$ vs control group (one-way ANOVA followed by Bonferroni's test). 
40 min. The expression intensity of Bax and Bcl-2 mRNA is reported as the ratio of the photodensities of $\mathrm{Bax}$ and $\mathrm{Bcl}-2$ to that of GAPDH.

\section{Statistical analysis}

Data were analyzed using the SPSS 17.0 software (IBM, USA). Results are reported as means \pm SD for each group and evaluated by one-way ANOVA. $\mathrm{P}<0.05$ was considered to be statistically significant.

\section{Results}

\section{Esculetin inhibited growth of hepatocellular carcinoma both in vivo and in vitro}

Cells were exposed to various concentrations of esculetin for 24,48 , or $72 \mathrm{~h}$ and cell proliferation was determined by MTT assay. Esculetin significantly inhibited the viability of hepatocellular carcinoma cells $(P<0.05)$ in a doseand time-dependent manner (Figure $1 \mathrm{~A})$. The $\mathrm{IC}_{50}$ values of esculetin against SMMC-7721 cells at $72 \mathrm{~h}$ was $2.24 \mathrm{mM}$. We also evaluated the antitumor effect of esculetin in vivo by establishing a xenograft tumor model in C57BL/6J mice by injection of Hepa1-6 cells. As shown in Figure 1B, the tumor inhibition rates of esculetin $\left(200,400\right.$, and $700 \mathrm{mg} \cdot \mathrm{kg}^{-1} \cdot$ day $\left.^{-1}\right)$ were $20.33,40.37$, and $55.42 \%$, respectively. Of note, even the highest dose of esculetin had no evident effect on the body weight of xenograft mice. Esculetin did not exhibit toxicity in this animal study. Taken together, our findings suggested that esculetin suppressed the growth of hepatocellular carcinoma both in vitro and in vivo (Figure 1).
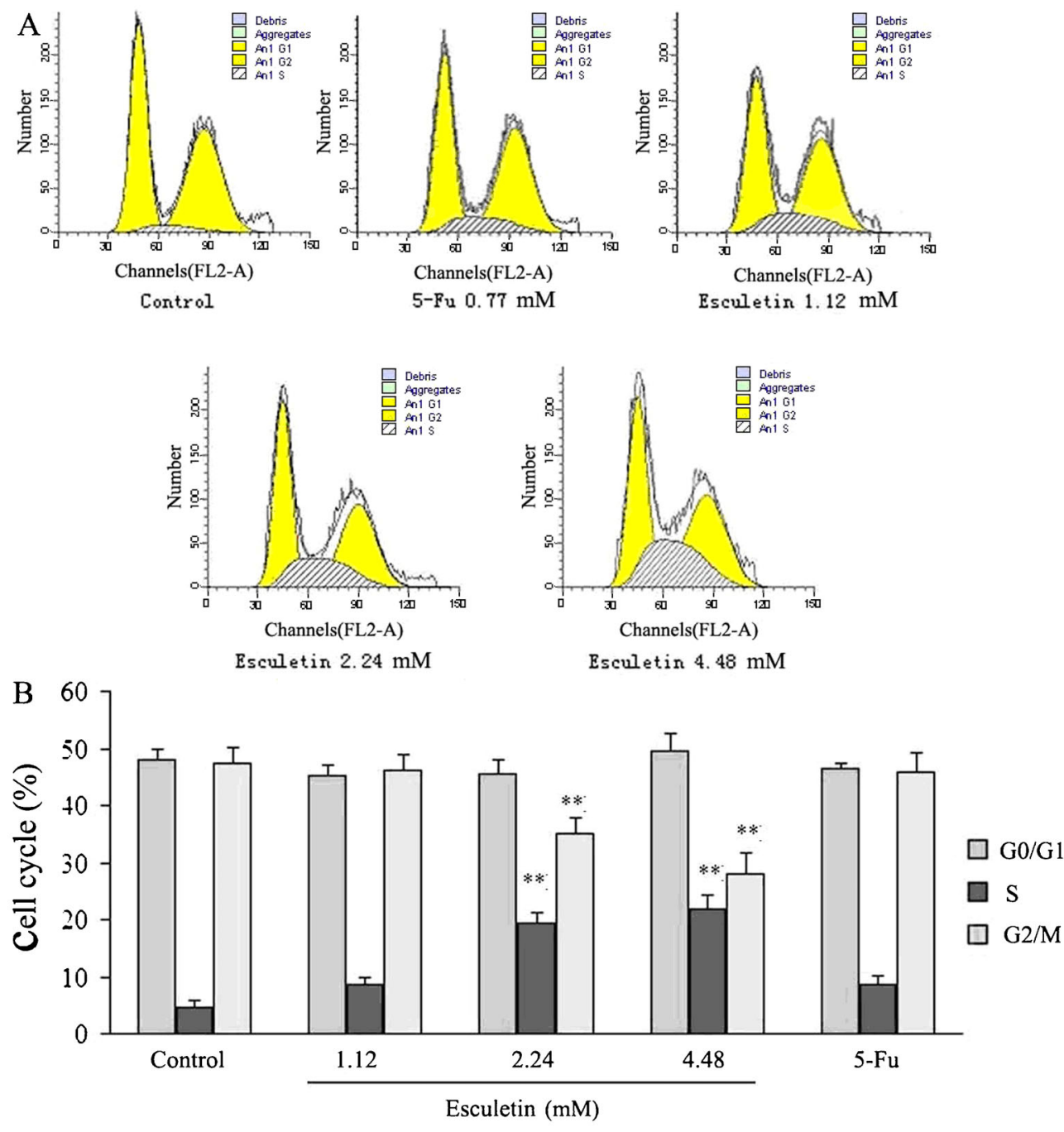

Figure 2. Effects of esculetin on the cell cycle of SMMC-7721. SMMC- 7721 cells were seeded at a density of $1 \times 10^{5} / \mathrm{mL}$ cells per culture flask for $24 \mathrm{~h}$, and harvested after treatment with esculetin $(0,1.12,2.24$, and $4.48 \mathrm{mM})$ and 5-FU (0.77 mM) for $48 \mathrm{~h}$. Cell cycle distributions in SMMC-7721 cells were determined by PI staining and flow cytometry analysis. Data were analyzed using the Bioconsort software and are reported as means $\pm \mathrm{SD}$. ${ }^{* *} \mathrm{P}<0.01 \mathrm{vs}$ control group (one-way ANOVA followed by Bonferroni's test). 

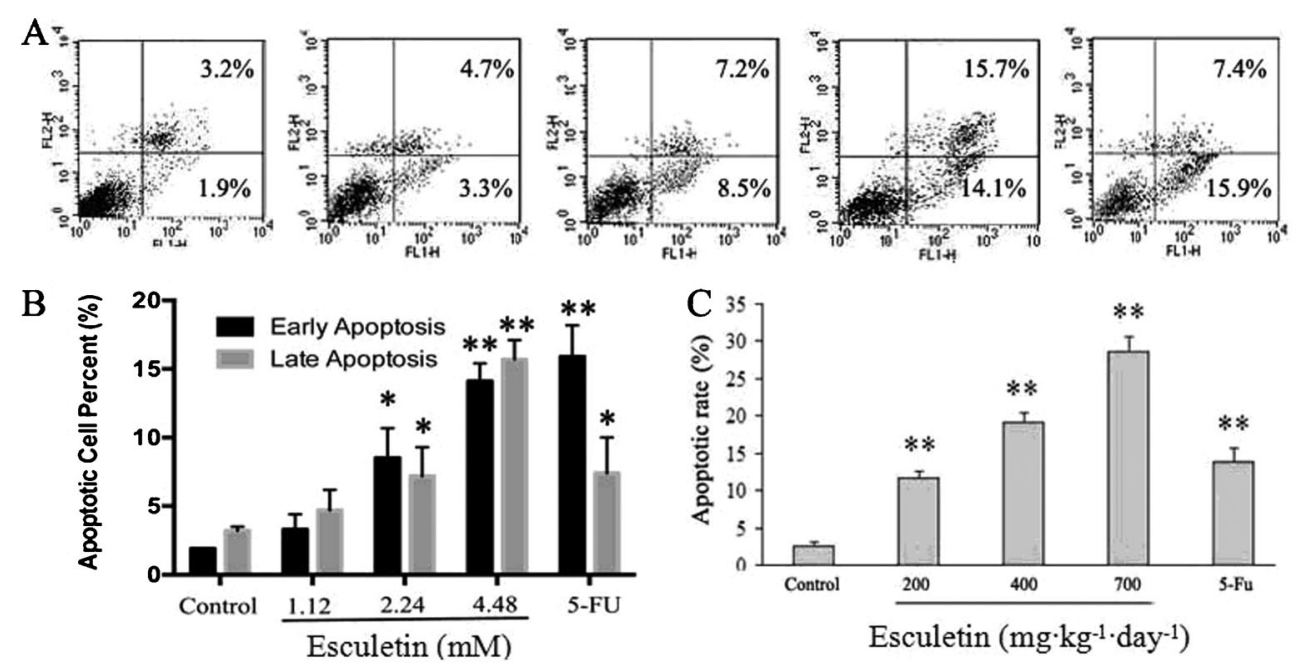

Figure 3. Esculetin induced apoptosis of hepatocellular carcinoma (HCC). SMMC-7721 cells were grouped and harvested after esculetin $(0,1.12,2.24$, and $4.48 \mathrm{mM})$ and $5-\mathrm{FU}(0.77 \mathrm{mM})$ treatment for $48 \mathrm{~h}(A$ and $B)$. Early and late apoptosis of HCC cells was determined by flow cytometry analysis using a PI-Annexin V-FITC kit. Data were analyzed using Bioconsort software and are reported as means $\pm S D$. In addition, apoptotic cells $(C)$ in tumors were also detected and analyzed using the Bioconsort software and are reported as means $\pm S D$ for $n=15$. ${ }^{*} P<0.01$ vs control group (one-way ANOVA followed by Bonferroni's test).

\section{Effects of esculetin on the cell cycle distribution in SMMC-7721 cells}

The in vitro and in vivo assays of esculetin antiproliferative activity revealed effects on cell-cycle distribution. SMMC-7721 cells were incubated with esculetin for $48 \mathrm{~h}$ and cell cycle distribution was assayed by FACS. As seen in Figure 2, esculetin at 2.24 and $4.48 \mathrm{mM}$ significantly increased the percentage of cells in S phase from $4.7 \%$ to 19.5 , and $22.2 \%$, respectively $(P<0.05)$. In addition, the percentages of cells in $\mathrm{G} 2 / \mathrm{M}$ phase decreased from $47.3 \%$ (control) to 35.0 and $28.3 \%$, respectively $(P<0.01$; Figure 2). These results suggest that esculetin blocked the cell cycle at S phase.

\section{Esculetin induced apoptosis in tumor and SMMC-7721 cells}

Apoptosis of cells was analyzed by flow cytometry using PI-Annexin V-FITC. As shown in Figure 3A and B, after treatment with esculetin and $5-\mathrm{Fu}$, the early and late apoptotic cells increased significantly in a dose-dependent manner. Apoptosis levels were 13.9, 19.1, 28.6, and 14.7\% when C57BL/6J mice were treated with low-, medium-, or high-dose $\left(200,400\right.$, or $700 \mathrm{mg} \cdot \mathrm{kg}^{-1} \cdot$ day $\left.^{-1}\right)$ esculetin, and $5-\mathrm{Fu}$, respectively $(\mathrm{P}<0.05)$. Esculetin treatment was thus consistently shown to induce apoptosis both in vivo and in vitro.

\section{Esculetin led to the loss of MMP in vivo and in vitro}

The effect of esculetin on MMP was examined in vivo and in vitro by JC-1 staining followed by FACS analysis. Intact mitochondrial membranes allow accumulation of JC-1 in the mitochondria, in which it will generate red fluorescence. Once loss of MMP occurs, JC-1 cannot accumulate in the mitochondria where it will remain as a monomer in the cytosol and fluoresce green light (14). Therefore, collapse of mitochondrial potential during apoptosis is indicated by an increase in the ratio of green to red fluorescence. When tumor-bearing C57BL/6J mice were treated with esculetin for 15 consecutive days, the MMP of the tumor tissues was significantly decreased compared with that of untreated animals $(P<0.01)$. The green fluorescence ratio in low-, medium-, or high-dose treatment groups was $17.5,36.7$, and $48.5 \%$, respectively, significantly higher than that of the untreated group. Exposure of cells to esculetin $(0,1.12,2.24$, or $4.48 \mathrm{mM})$ for $48 \mathrm{~h}$ led to significant decreases of MMP level. This result suggests that esculetin caused the loss of mitochondrial membrane potential in HCC in a dose-dependent manner (Figure 4).

\section{Esculetin induced activation of caspase-3, -8, and -9 in SMMC-7721 cells}

Caspases are known to be a pivotal regulator in the process of cellular apoptosis (15). Thus, we investigated effects of esculetin on the activation of caspase- $3,-8$, and -9 by a colorimetric assay. We found that esculetin significantly induced activation of caspase- 9 and -3 in SMMC7721 cells $(\mathrm{P}<0.05$ vs untreated cells), but did not affect caspase- 8 activity. The results indicated that esculetin treatment induced apoptosis in SMMC-7721 cells through an intrinsic apoptosis pathway (Figure 5).

\section{Effect of esculetin on Bcl-2 and Bax expression}

$\mathrm{Bcl}-2$ family proteins play an important role in the 

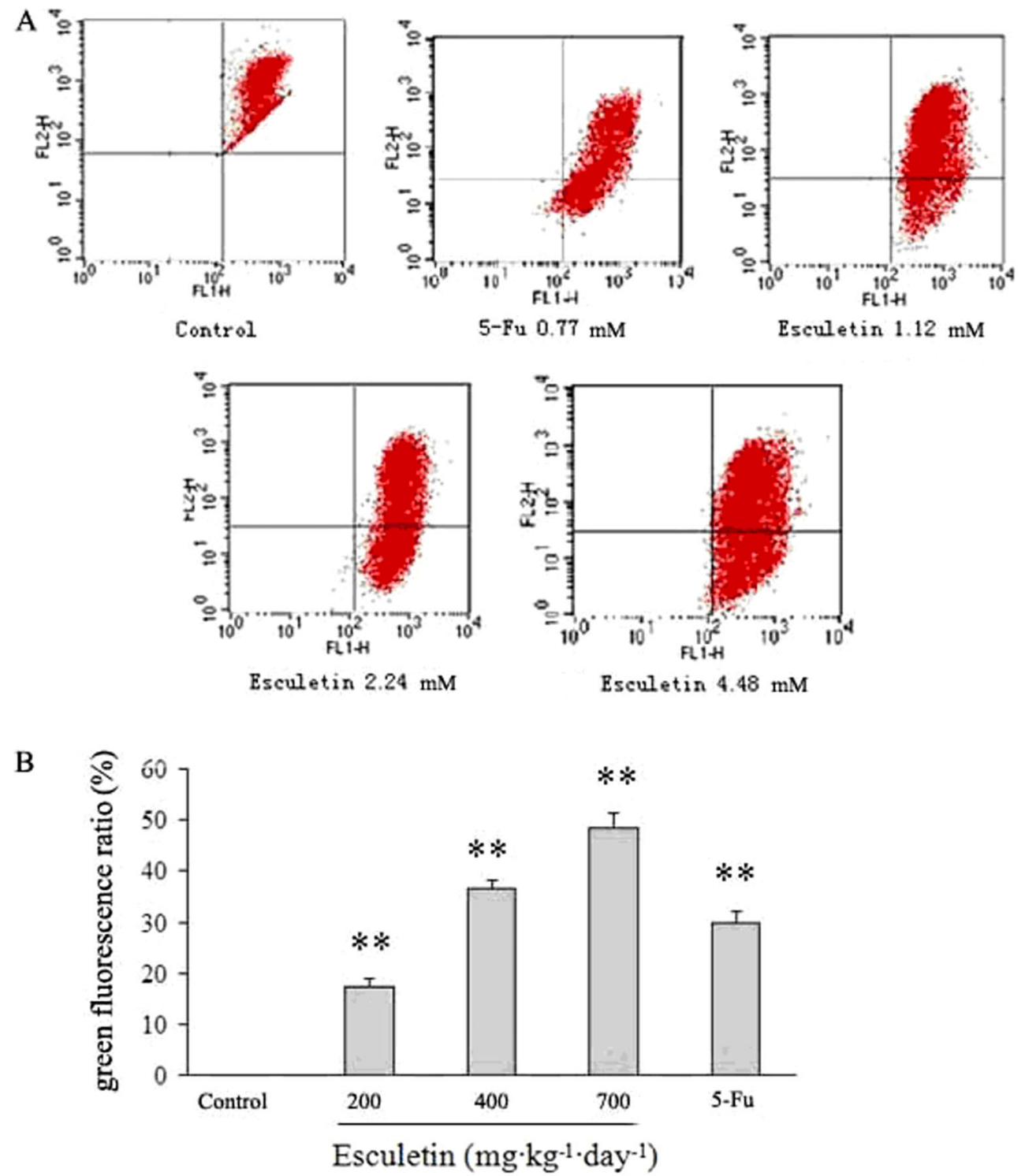

Figure 4. Esculetin caused loss of mitochondrial potential in SMMC-7721 cells $(A)$ and in vivo $(B)$. Mitochondrial membrane potential (MMP, $\Delta \Psi \mathrm{m}$ ) was determined by flow cytometry analysis with JC-1 staining. Data were analyzed using the Bioconsort software and are reported as mean $\pm S D$ for $n=15$. ${ }^{* *} P<0.01$ vs control group (one-way ANOVA followed by Bonferroni's test).

mitochondria-mediated apoptosis pathway, including Bax and Bcl-2 (16). To further understand the molecular mechanism of esculetin-mediated cellular apoptosis, expression of $\mathrm{Bcl}-2$ and Bax was evaluated. As shown in Figure $6 \mathrm{~A}$ and $\mathrm{B}$, expression of $\mathrm{Bcl}-2$ and $\mathrm{Bax}$ were significantly changed after esculetin treatment $(P<0.01)$. Esculetin resulted in an increase of Bax (proapoptotic) protein expression in a dosedependent manner and a decrease of $\mathrm{Bcl}-2$ (antiapoptotic) protein expression in tumors of both $\mathrm{C} 57 \mathrm{BL} / 6 \mathrm{~J}$ mice and SMMC-7721 cells. These results suggest that esculetin caused an increase of the $\mathrm{Bax} / \mathrm{Bcl}-2$ ratio that might be involved in mitochondria-dependent apoptosis.
In addition, expression of $\mathrm{Bcl}-2$ and $\mathrm{Bax}$ in $\mathrm{C} 57 \mathrm{BL} / 6 \mathrm{~J}$ mice was assayed by RT-PCR. As shown in Figure 6C, expression of $\mathrm{Bcl}-2$ and $\mathrm{Bax}$ mRNA was consistent with expression of the corresponding proteins. Treatment with esculetin resulted in a significant decrease of antiapoptotic Bcl-2 mRNA levels in Hepa1-6 tumors, whereas that of proapoptotic Bax was significantly increased $(P<0.05)$.

\section{Discussion}

Recently, several groups reported that esculetin had antitumor activity in many types of cancer. For instance, 


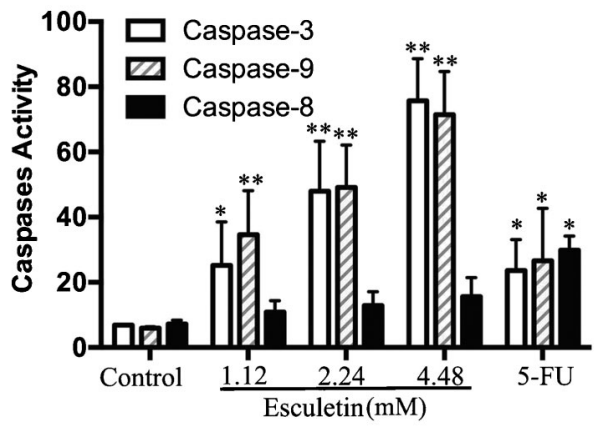

Figure 5. Effect of esculetin on the activity of caspases in SMMC7721 cells. SMMC-7721 cells were grouped and harvested after esculetin $(0,1.12,2.24$, and $4.48 \mathrm{mM})$ and 5 -FU $(0.77 \mathrm{mM})$ treatment for $48 \mathrm{~h}$. The caspase activities induced by esculetin were determined by colorimetric assays using caspase- $3,-9$, and -8 activation kits, following the manufacturer's instructions. All measurements were performed 3 times and the activity of caspases was determined by measuring changes in absorbance at $405 \mathrm{~nm}$ using the ELISA reader. Data are reported as means $\pm S D$ for 6 in each group. ${ }^{*} \mathrm{P}<0.05$, ${ }^{* *} \mathrm{P}<0.01$ vs control group (one-way ANOVA followed by Bonferroni's test).

esculetin induced apoptosis of human NB4 acute promyelocytic leukemia cells (17) and also initiated apoptosis in U937 human leukemia cells through activation of JNK and ERK (9). Although several in vitro studies have demonstrated the inhibition of cancer cell proliferation by esculetin, there are few in vivo studies of its therapeutic effect in HCC. This study evaluated the antitumor activity of esculetin by its effects on cell proliferation and tumor growth in a C57BL/6J mouse xenograft model. Our data showed that esculetin significantly inhibited the growth of HCC. Of note, esculetin had no obvious toxicity in this animal study.

Based on the inhibitory effect of esculetin on growth of HCC, the potential mechanism of esculetin action was studied. Apoptosis is a key process mediated by chemotherapeutic agents and it contributes to efficacy (18). Esculetininduced apoptosis has been reported in other types of cancer including human colon cancer, human oral cancer, and lung cancer $(9,19-21)$. Herein, our results showed that esculetin could effectively induce apoptosis in both tumorbearing mice and SMMC-7721 cells. The apoptotic rate reached $55.42 \%$ after treatment with high-dose esculetin in vivo. In addition, we also investigated the effect of esculetin on the cell cycle. Esculetin treatment caused S-phase arrest in SMMC-7721 cells. These results demonstrated that esculetin inhibited tumor cell growth by arresting the cell cycle in S phase and inducing apoptosis.

Apoptosis-signaling cascades can be divided into two major pathways: a death-receptor-induced extrinsic pathway and a mitochondria-apoptosome-mediated intrinsic pathway (22). Apoptosis is modulated by active caspases that are derived from inactive zymogens in a cascade of sequential cleavage reactions (23). Caspase- 8 is involved in the death-receptor-induced extrinsic apoptosis pathway
A

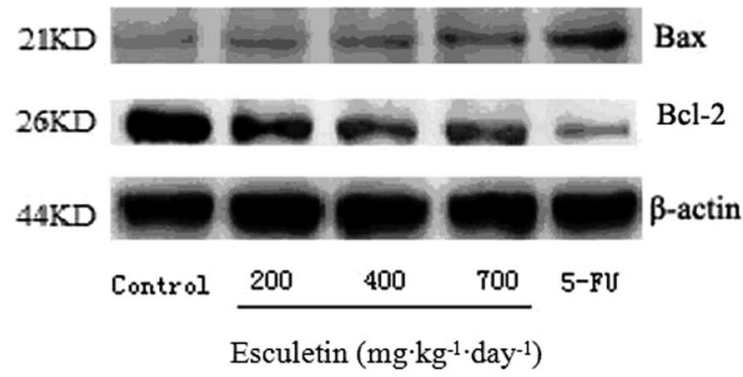

B

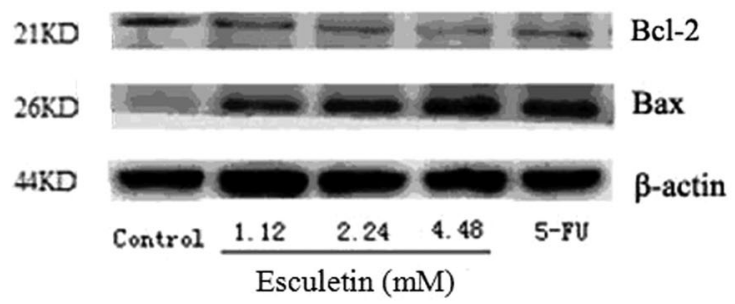

C

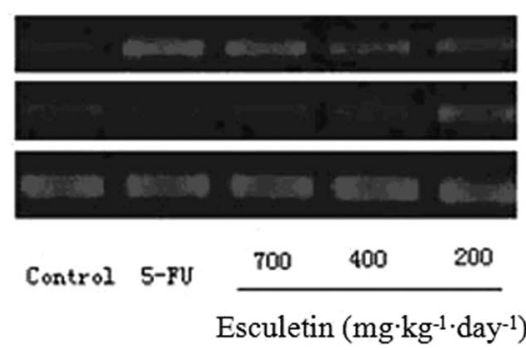

Bax

Bcl-2

GAPDH

Figure 6. Effect of esculetin on the expression of $\mathrm{Bax}$ and $\mathrm{Bcl}-2$. The protein expression of $\mathrm{Bcl}-2$ and $\mathrm{Bax}$ was determined by Western blot analysis $(A$ and $B)$. The mRNA expression of Bax and $\mathrm{Bcl}-2$ was detected by RT-PCR $(C)$.

while caspase- 9 is associated with the mitochondria apoptotic pathway $(24,25)$. Caspase- 9 is the initiator of the mitochondria apoptotic pathway, which is activated in a complex termed the apoptosome by the scaffold protein Apaf- 1 and its cofactor cytochrome C. Cytochrome $\mathrm{C}$ acts in association with other cytosolic factors to cause activation of executioner caspase-3, in turn leading to downstream apoptotic events (26). In this study, our results demonstrated that esculetin induced apoptosis through the activation and cleavage of capase- 3 and -9 .

The Bcl-2 family includes regulators of the mitochondria-mediated apoptosis pathway, such as Bax and Bcl-2 (27). Following an apoptosis signal, the proapoptotic protein Bax translocates to the outer mitochondrial membrane, promoting permeabilization and the release of various apoptotic factors. Antiapoptotic Bcl-2 has been shown to prevent apoptosis by forming a heterodimer with a proapoptotic member, such as Bax, resulting in neutralization of proapoptotic effects. Bcl-2 can also influence the permeability of the intracellular membranes of mitochondria 
and the activation of caspase-3 (28). Therefore, the balance of the expression of $\mathrm{Bcl}-2$ and Bax proteins is crucial for cell survival and cell death $(29,30)$. In this investigation, we found that Bax expression was significantly elevated while $\mathrm{Bcl}-2$ expression was significantly decreased both in vitro and in vivo. Consistent with this, the transcriptional levels of Bax and Bcl-2 mRNA were changed in Hepa1-6 tumors after esculetin treatment, which ultimately resulted in an increase in the $\mathrm{Bax} / \mathrm{Bcl}-2$ ratio and activation of the caspase cascade (Figure 6). In addition, our results showed that esculetin treatment induced loss of MMP. Therefore, esculetin induced apoptosis in HCC through the mitochondria-mediated intrinsic pathway. Taken together, our findings suggest that cellular apoptosis mediated in HCC by esculetin in vivo and vitro was dependent on caspase- 9 and

\section{References}

1. Jemal A, Bray F, Center MM, Ferlay J, Ward E, Forman D. Global cancer statistics. CA Cancer J Clin 2011; 61: 69-90, doi: 10.3322/caac.20107.

2. Han KH, Park JY. Systemic treatment in advanced/metastatic hepatocellular carcinoma in the era of targeted therapy. J Gastroenterol Hepatol 2010; 25: 1023-1025, doi: 10.1111/ j.1440-1746.2010.06359.x

3. Levin B, Amos C. Therapy of unresectable hepatocellular carcinoma. N Engl J Med 1995; 332: 1294-1296, doi: 10.1056/ NEJM199505113321910.

4. Hecht SS, Kenney PM, Wang M, Trushin N, Agarwal S, Rao $\mathrm{AV}$, et al. Evaluation of butylated hydroxyanisole, myoinositol, curcumin, esculetin, resveratrol and lycopene as inhibitors of benzo[a]pyrene plus 4-(methylnitrosamino)-1-(3pyridyl)-1-butanone-induced lung tumorigenesis in $\mathrm{A} / \mathrm{J}$ mice. Cancer Lett 1999; 137: 123-130, doi: 10.1016/S0304-3835(98) 00326-7.

5. Witaicenis A, Seito LN, Di Stasi LC. Intestinal anti-inflammatory activity of esculetin and 4-methylesculetin in the trinitrobenzenesulphonic acid model of rat colitis. Chem Biol Interact 2010; 186: 211-218, doi: 10.1016/j.cbi.2010.03.045.

6. Yang J, Xiao YL, He XR, Qiu GF, Hu XM. Aesculetin-induced apoptosis through a ROS-mediated mitochondrial dysfunction pathway in human cervical cancer cells. J Asian Nat Prod Res 2010; 12: 185-193, doi: 10.1080/10286020903427336.

7. Subramaniam SR, Ellis EM. Neuroprotective effects of umbelliferone and esculetin in a mouse model of Parkinson's disease. J Neurosci Res 2013; 91: 453-461, doi: 10.1002/ jnr.23164.

8. Pan SL, Huang YW, Guh JH, Chang YL, Peng CY, Teng CM. Esculetin inhibits Ras-mediated cell proliferation and attenuates vascular restenosis following angioplasty in rats. Biochem Pharmacol 2003; 65: 1897-1905, doi: 10.1016/S0006-2952(03) 00161-8.

9. Park SS, Park SK, Lim JH, Choi YH, Kim WJ, Moon SK. Esculetin inhibits cell proliferation through the Ras/ERK1/2 pathway in human colon cancer cells. Oncol Rep 2011; 25: 223-230.

10. Park C, Jin CY, Kim GY, Choi IW, Kwon TK, Choi BT, et al. Induction of apoptosis by esculetin in human leukemia U937 cells through activation of JNK and ERK. Toxicol Appl
-3 and Bcl-2 family proteins.

In summary, in vitro and in vivo antitumor effects in human HCC were associated with S-phase arrest and apoptosis. In addition, esculetin elevated the $\mathrm{Bax} / \mathrm{Bcl}-2$ ratio, activated caspase- 3 and -9 , and induced the mitochondrialmediated apoptosis pathway in HCC cells. The present study provides evidence supporting esculetin as a potential therapeutic agent for the treatment of hepatocellular carcinoma.

\section{Acknowledgments}

Research supported by National Natural Science Funds of China (Grant \#81202556) and Natural Science Foundation of Liaoning Province (Grant \#20130222063).

Pharmacol 2008; 227: 219-228, doi: 10.1016/j.taap.2007. 10.003.

11. Kuo HC, Lee HJ, Hu CC, Shun HI, Tseng TH. Enhancement of esculetin on Taxol-induced apoptosis in human hepatoma HepG2 cells. Toxicol Appl Pharmacol 2006; 210: 55-62, doi: 10.1016/j.taap.2005.06.020.

12. Jing Wang, Hong-yu Li, Hong-Xin Wang. The separation and purification technology of esculetin from CORTEX FRAXINI. Chinese Traditional Patent Med 2009; 31: 294-296.

13. Wei N, Liu GT, Chen XG, Liu Q, Wang FP, Sun H. H1, a derivative of Tetrandrine, exerts anti-MDR activity by initiating intrinsic apoptosis pathway and inhibiting the activation of Erk1/2 and Akt1/2. Biochem Pharmacol 2011; 82: 15931603, doi: 10.1016/j.bcp.2011.08.012.

14. Yokosuka $T$, Goto $H$, Fujii $H$, Naruto $T$, Takeuchi $M$, Tanoshima R, et al. Flow cytometric chemosensitivity assay using JC1, a sensor of mitochondrial transmembrane potential, in acute leukemia. Cancer Chemother Pharmacol 2013; 72: 1335-1342, doi: 10.1007/s00280-013-2303-x.

15. Lavrik IN, Golks A, Krammer PH. Caspases: pharmacological manipulation of cell death. J Clin Invest 2005; 115: 2665-2672, doi: 10.1172/JCl26252.

16. Jurgensmeier JM, Xie Z, Deveraux Q, Ellerby L, Bredesen D, Reed JC. Bax directly induces release of cytochrome C from isolated mitochondria. Proc Natl Acad Sci U S A 1998; 95: 4997-5002, doi: 10.1073/pnas.95.9.4997.

17. Rubio V, Calvino E, Garcia-Perez A, Herraez A, Diez JC. Human acute promyelocytic leukemia NB4 cells are sensitive to esculetin through induction of an apoptotic mechanism. Chem Biol Interact 2014; 220C: 129-139, doi: 10.1016/j.cbi. 2014.06.021.

18. Holohan C, Van Schaeybroeck S, Longley DB, Johnston PG. Cancer drug resistance: an evolving paradigm. Nat Rev Cancer 2013; 13: 714-726, doi: 10.1038/nrc3599.

19. Lee SY, Lim TG, Chen H, Jung SK, Lee HJ, Lee MH, et al. Esculetin suppresses proliferation of human colon cancer cells by directly targeting beta-catenin. Cancer Prev Res 2013; 6: 1356-1364, doi: 10.1158/1940-6207.CAPR-13-0241.

20. Kok SH, Yeh CC, Chen ML, Kuo MY. Esculetin enhances TRAIL-induced apoptosis through DR5 upregulation in human oral cancer SAS cells. Oral Oncol 2009; 45: 1067-1072, 
doi: 10.1016/j.oraloncology.2009.07.018.

21. Lacy A, O'Kennedy R. Studies on coumarins and coumarinrelated compounds to determine their therapeutic role in the treatment of cancer. Curr Pharm Des 2004; 10: 3797-3811, doi: 10.2174/1381612043382693.

22. Hu W, Kavanagh JJ. Anticancer therapy targeting the apoptotic pathway. Lancet Oncol 2003; 4: 721-729, doi: 10.1016/S1470-2045(03)01277-4.

23. Cullen SP, Martin SJ. Caspase activation pathways: some recent progress. Cell Death Differ 2009; 16: 935-938, doi: 10.1038/cdd.2009.59.

24. Li J, Yuan J. Caspases in apoptosis and beyond. Oncogene 2008; 27: 6194-6206, doi: 10.1038/onc.2008.297.

25. Kumar S. Caspase function in programmed cell death. Cell Death Differ 2007; 14: 32-43, doi: 10.1038/sj.cdd.4402060.

26. Jiang X, Wang X. Cytochrome C-mediated apoptosis. Annu
Rev Biochem 2004; 73: 87-106, doi: 10.1146/annurev. biochem.73.011303.073706.

27. Czabotar PE, Lessene G, Strasser A, Adams JM. Control of apoptosis by the BCL-2 protein family: implications for physiology and therapy. Nat Rev Mol Cell Biol 2014; 15: 4963, doi: 10.1038/nrm3722.

28. Pastorino JG, Chen ST, Tafani M, Snyder JW, Farber JL. The overexpression of Bax produces cell death upon induction of the mitochondrial permeability transition. J Biol Chem 1998; 273: 7770-7775, doi: 10.1074/jbc.273.13.7770.

29. Low IC, Kang J, Pervaiz S. Bcl-2: a prime regulator of mitochondrial redox metabolism in cancer cells. Antioxid Redox Signal 2011; 15: 2975-2987, doi: 10.1089/ars.2010.3851.

30. Wong WW, Puthalakath $\mathrm{H}$. Bcl-2 family proteins: the sentinels of the mitochondrial apoptosis pathway. IUBMB Life 2008; 60: 390-397, doi: 10.1002/iub.51. 